analecta polit. | Vol. 7 | No. 13 | PP. 357-397

| julio-diciembre | 2017 | ISSN: 2027-7458 | Medellín- Colombia

http://dx.doi.org/10.18566/apolit.v7n13.a08

\section{La Fédération} de Russie est-elle une grande puissance (1991-2001)?

Is the Russian Federation a Great Power (1991-2001)?

\section{É a Federação Russa uma grande potência (1991-2001)?}

\section{CHRISTIAN CAMILO CASAS GARCÍA}

este artículo en APA:

Casas, C. (2017). La

Fedérération de Russie

et-elle une grande

puissance (1991-2001)?

Analecta Política, 7(13),

357-397

Recibido:

10 de diciembre de

2016

Aprobado:

15 de agosto de 2016

\begin{abstract}
Master en Histoire de l'Université Paris-Sorbonne IV, professeur et chercheur de la même université. Dynamique des systèmes internationaux : négocier, communiquer, entreprendre à l'époque moderne et contemporaine. Correo : christian.casas_garcia@ paris-sorbonne.fr. orcid.org/0000-0003-1515-2026. Direccion postal : 75116. Paris - Francia
\end{abstract}




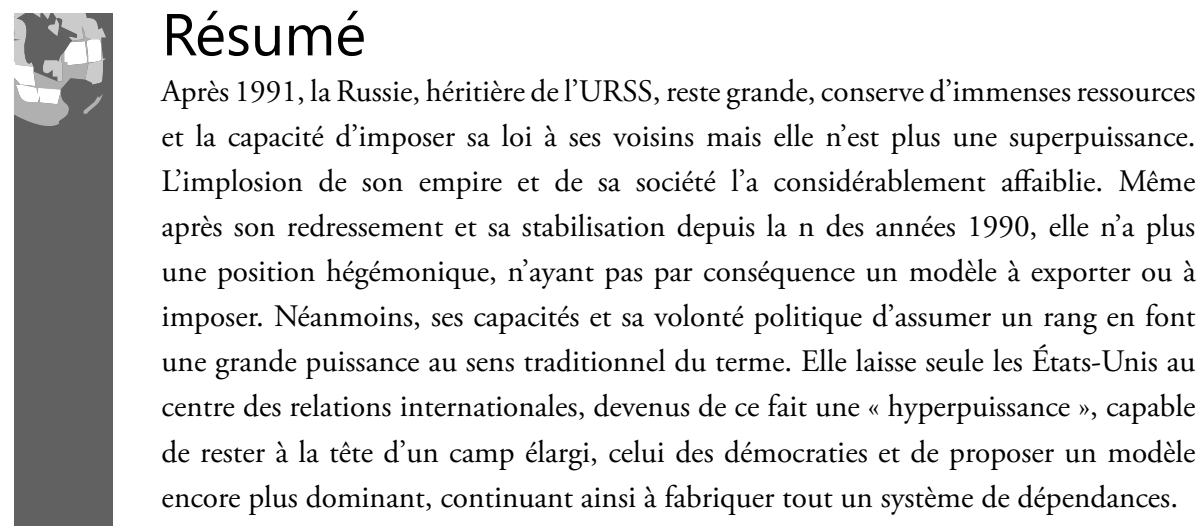

\section{Mots-clés}

Système international post-soviétique, puissance, représentation, Russie, redressement.

\section{Resumen}

Después de 1991, Rusia, heredera de la Unión Soviética, es todavía un Estado importante en el sistema internacional, que conserva numerosos recursos naturales y humanos. Esto le da la posibilidad de imponer aún su ley a sus vecinos próximos, pero ya no es una superpotencia; la crisis de su imperio y de su sociedad la debilitaron considerablemente. Incluso después de la recuperación y estabilización económica que tuvo lugar desde 1990, este Estado ya no posee una posición hegemónica, lo que le impide importar o imponer un modelo socio-económico y político a seguir. Pese a esta situación, sus capacidades y su voluntad política de asumir un rango en los asuntos mundiales, hacen de ella una "gran potencia" en el sentido tradicional del término. De esta manera, Rusia le cede una posición central en las relaciones internacionales a los Estados Unidos, resultando de este hecho una "hiperpotencia", lo cual le permite a Washington liderar un campo bastante extenso, el de las democracias y proponer un modelo mucho más dominante. La presente contribución analiza la situación de Rusia bajo la administración de Boris Yeltsin y el comienzo de la presidencia de Vladímir Putin, marcados por la construcción de un Estado fuerte y próspero, tanto internamente, como en el exterior.

\section{Palabras clave}

Sistema internacional post-soviético, potencia, representación; Rusia, recuperación. 


\section{Abstract}

After 1991, Russia, heir to the Soviet Union, remains a strong State within the world order and maintains considerable human and natural resources. This situation allows Russia to impose her will over some of the neighboring States. However, Russia cannot longer be considered a superpower: the crisis of her rule and society debilitated her. Even after the economic recovery and stabilization, which began in 1990, this State no longer has a dominant position to impose a socio-economic or political model. Despite the aforementioned situation, Russias capability and political will to have an influential position within international politics make of her a "Great Power" in the traditional sense of the word. Consequently, Russia leaves a privileged position within international relations to the United States of America, making of the latter a "hyperpower". Such a situation allows Washington to exercise her leadership over a significant number of States -those ruled by democracies- and to pose a more dominant model. Bearing this in mind, the article analyzes the situation of Russia during the administration of Boris Yeltsin and the beginning of the presidency of Vladimir Putin, periods known for the efforts to consolidate a strong and prosperous State, both internally and in relation to other countries.

\section{Key words}

Post-Soviet World Order, great powers, representation, Russia, recovery.

\section{Resumo}

Depois de 1991, a Rússia, herdeira da União Soviética, continua a ser um Estado importante no sistema internacional, que conserva numerosos recursos naturais e humanos. Isto lhe dá a possibilidade de impor sua lei aos vizinhos, mas não é mais uma superpotência; A crise do seu império e a sua sociedade, a enfraqueceram consideravelmente. Mesmo após a recuperação e a estabilização econômica desde 1990, o estado Russo não tem mais uma posição hegemônica, o que impede a importação ou a imposição de um modelo socioeconômico e político a seguir. Apesar desta situação, suas capacidades e sua vontade política de assumir uma posição relevante nos assuntos mundiais, fazem dela uma "grande potência» no sentido tradicional do termo. Desta forma, a Rússia cede uma posição central nas relações internacionais aos Estados Unidos, resultando, deste fato uma "hiperpotência», o que permite que Washington liderar um campo bastante extenso, aquele das democracias, e propor um modelo ainda mais dominante. A presente contribuição analisa a situação da Rússia sob a administraçáo de Boris Yeltsin, e o início da presidência de Vladimir Putin, marcados pela construção de um Estado forte e próspero, tanto no interior, como no exterior.

\section{Palavras-Chave}

Sistema internacional pos-soviético, potência, representação, Rússia, recuperação 
« Il y a trois moyens d'agir sur les hommes a Vladimir Poutine un jour déclaré sur un ton badin : le chantage, la vodka et la menace d'assassinat " (Vaïsse, 2013, p.243). Pour Poutine, la parole n'exprime pas que des pensées ou des sentiments, elle est un pur instrument servant à se rendre maitre de la volonté d'autrui, à convaincre l'interlocuteur ou bien une couverture visant à tromper l'ennemi, qui utilise la démocratie comme un masque pour faire main basse sur ses richesses. Ainsi s'explique l'un des paradoxes poutiniens qui a laissé les observateurs perplexes concernant sa politique étrangère : sa capacité de dire une chose un jour et de faire son contraire quelques jours plus tard.

Dans un article publié en 1979, Jean-Baptiste Duroselle en analysant le concept de "grande puissance " la définit comme un État capable d'assurer à lui seul sa sécurité contre toute autre puissance. Cette définition met en avant une capacité structurelle, indépendante du jeu des victoires et des échecs.

Si bien les liens étroits entre puissance et sécurité sont importants, il convient d'élargir la définition en prenant en compte la variété des composantes du hard et du soft power (Frank, 2012). Tous les historiens s'accordent sur un point : plus une puissance est grande, plus elle est capable de disposer d'un ensemble de possibilités et de moyens d'action à l'extérieur dans les domaines les plus différenciés, plus elle peut également jouer sur une palette variée de méthodes pour imposer sa volonté. On peut souligner notamment la peur et dissuasion, ou la persuasion et l'attachement par l'aide économique, le rayonnement, l'adhésion à ses idées ou à son modèle politique et social.

Le 25 décembre 1991, la RSFSR change d'appellation et devient la Fédération de Russie. Pour la première fois dans son histoire en tant qu'État moderne, la Russie, en surmontant des difficultés diverses sur le plan social, économique, politique et identitaire s'est engagée dans un long et difficile processus de sortie du communisme en tant que système politique et économique. La restauration de l'autorité de l'État dans un contexte d'importantes mutations systématiques représente l'un des grands défis de la fin de décennie post-soviétique.

Au tournant du sicle, on se trouve à l'aube d'un monde nouveau, la plupart des fondements de la deuxième moitié du $\mathrm{XX}^{\mathrm{e}}$ siècle ont disparus ou sont dévalorisés. On constate une reprise de l'économie mondiale lente à se réveiller entre 1992 et 1993, favorisée par la baise des cours du pétrole. Mais de 1994 à 2000, on remarque une croissance remarquable des pays anglo-saxons et l'émergence de nouveaux pays industrialisés, en particulier asiatiques. 
La mondialisation était en marche avec l'expansion du libre échange aux anciens pays d'économie collectiviste, l'adoption généralisée des lois du marché, l'explosion de la nouvelle économie et la tendance à la mobilité des capitaux (Vaïsse, 2013). Cela a eu comme conséquence une interdépendance accrue des États, sans pour autant qu'existent des moyens de régulation adaptés.

C'est également une période d'émergence des acteurs non étatiques et transnationaux, qui commencent à jouer un rôle de plus en plus important dans les relations internationales. Ce n'est pas un monde multipolaire qui succède à l'ancien monde bipolaire, mais un univers " chaotique " marqué par un Amérique à la fois hyperpuissante et limitée dans son efficacité, une Russie en période de redressement, une Asie gravement perturbée, une Afrique en proie aux troubles et un Moyen-Orient dans l'impasse (Chaliand, 2013).

L'objectif de notre analyse est de comprendre les moyens d'action et les méthodes qu’a utilisée la Fédération de Russie, pour maintenir son statut de grande puissance à l'intérieur et l'extérieure du pays, entre les deux mandants de Boris Nikolä̈evitch Eltsine entre 1990-1999 et les débuts de la présidence de Vladimir Poutine élu le 7 mai 2000. Ainsi, notre étude se centre dans le processus de restauration de l'hégémonie russe dans le monde. C'est pour cela qu'il est important de nous focaliser sur la période de redressement sous Eltsine jusqu'à l'entreprise lancée par Poutine de restauration de la puissance russe et sa rapide ascension sur la scène internationale.

Tout cela nous amène à nous demander dans quelle mesure la Fédération de Russie tout en étant une puissance affaiblie a réussi à s'imposer dans le système international post-soviétique?

Pour répondre à cette question notre étude va se structurer en trois parties. Tout d'abord on va analyser les attributs d'une grande puissance. Ensuite, il sera question d'étudier l'État en transition de Boris Eltsine. Finalement, on va démontrer l'affirmation d'un État fort lancée par l'administration de Vladimir Poutine.

\section{La Russie et les attributs de la puissance Un regard sur l'école des « forces profondes »}

Létude du rôle des " forces profondes " s'agissait en premier lieu d'un regard plus diversifié sur les facteurs qui placent l'État au centre de l'activité internationale, 
notamment les facteurs économiques (Frank, 2012). Jean-Baptiste Duroselle et Pierre Renouvin dans leur Introduction à l'histoire des relations internationales ont mis surtout l'accent sur le rôle des facteurs géographique et démographique, sans accorder toute l'importance au facteur économique et ce que ces facteurs pouvaient représenter en terme de puissance ou de vulnérabilité. Ainsi, il convient de s'attarder sur la question géographique en terme des ressources naturelles en Russie et de son poids démographique, qui la placerait au cœur des enjeux en tant qu'acteur de premier plan dans les relations internationales.

La Fédération de Russie s'étend sur $17171750 \mathrm{~km}^{2}$ et comptait avec 147290 300 millions d'habitants selon le recensement en 2002 (Wackermann, 2007). En raison de l'étendue du pays, l'éventail des ressources est considérable, utilisées pour satisfaire aux besoins et aux usages de la société, pour se nourrir, produire de l'énergie, fabriquer, construire et se déplacer. La Russie disposent notamment de très nombreuses ressources minérales dont certaines en grande quantité. Son territoire détient le tiers des réserves mondiales de gaz naturel, deux tiers des réserves de minerai de fer, près d'un cinquième des réserves de charbon. Ces ressources ont été et sont exploitées avec deux objectifs : d'une part servir de base matérielle à la croissance industrielle, d'autre part apporter des sources de financement.

Après la collectivisation soviétique, la privatisation post-soviétique posa la question de l'appropriation et de la maîtrise des ressources naturelles. C'est l'État qui accorde des concessions d'exploitation à des entreprises publiques ou privées. Les grandes entreprises mondiales du pétrole, du gaz et de la métallurgie sont intéressées par les ressources de la Russie qui deviennent une source de rivalités et des tensions géopolitiques entre les firmes, les États et les régions. La Russie fournisseur principal en matières premières, principalement en sources d'énergie de plusieurs États post-soviétiques et d'Europe centrale, utilise cette arme pour exécrer une pression sur eux (Thorez, 2007). Il faut souligner également les exploitations des minerais qui sont très nombreuses. Parmi les plus importants on compte le fer dont plus du quart des réserves mondiales se trouve en Russie, le cuivre, le nickel, le platine, le wolfram et le molybdène.

En outre, les ressources énergétiques ont permis à la Russie d'en devenir un exportateur de premier plan. Elle détient en effet près d'un tiers des réserves mondiales de gaz naturel, environ $7 \%$ des réserves de pétrole et $17 \%$ des réserves de charbon (Wackermann, 2007).

Cependant le pays possède des ressources agraires relativement modestes. Parmi elles figurent les sols, les ressources végétales et animales. Ainsi, les terres agricoles 
n'occupent que 220 millions d'hectares, soit $12,5 \%$ de la superficie totale (Thorez, 2007). Pour l'essentiel les sols sont peu favorables à l'exploitation agricole. La Russie n'est pas également très riche en espèces végétales et animales. C'est notamment les végétaux marins qui commencent, à partir de 1990 à être de plus en plus utilisés dans les industries alimentaires et pharmaceutiques. La pêche est une autre activité pratiquée intensément dans les eaux côtières poissonneuses de l'océan Pacifique et dans les eaux douces continentales où se développe la pisciculture.

La population de la Russie diminue depuis la fin de la RSFSR, car les apports de l'immigration n'ont pas pu compenser le déficit de la croissance naturelle, provoqué par le niveau élevé de la mortalité et la faiblisse de la natalité (Kortchagina, 2005). Les indicateurs démographiques se sont détériorés pendant la décennie 1990. Mais depuis 2000, la natalité connaît une timide reprise.

À la fin des années 1980, on constate une phase de baise démographique, qui s'explique principalement par une baisse sensible du taux de fécondité. Cette diminution de la natalité est en relation avec la crise sociale et les incertitudes qui caractérisent la dernière décennie du XXe siècle et le début du XXI $\mathrm{X}^{\mathrm{e}}$. A contrario, la stabilisation économique, politique et sociale à partir des années 2000 , ainsi que le renforcement d'une nouvelle classe moyenne émergeante favorisent le développement du pays (Wackermann, 2007).

Le déchirement de la société russe après l'effondrement de l'URSS a considérablement affaibli la puissance russe, et a empêché ce pays de se montrer comme un véritable État puissant, malgré sa volonté de représentation en position de force.

\section{Les moyens de la Fédération de Russie pour s'imposer dans la scène mondiale : l'antiaméricanisme et le projet d'une union eurasienne}

C'est dans le deuxième chapitre de l'Introduction à l'histoire des relations internationales rédigé par Pierre Renouvin, qu'il réalise une analyse des " facteurs géographiques» (Renouvin, 1991). Il s'interroge sur la place qu'il convient d'accorder à " l'espace ", c'est-à-dire à "l'étendue du territoire qui occupe l'État et ses influences sur les États périphériques » et de leur impact sur la vie des États et la 
vie internationale. En d'autres termes, la représentation de la puissance à l'intérieur et à l'extérieur du territoire. Un grand territoire, de nombreuses ressources naturelles, une forte population ne donnent pas nécessairement de la puissance. À l'inverse, un État dépourvu de richesses du sol et du sous-sol peut devenir un pays puissant dans le monde.

Il convient d'analyser la volonté russe d'avoir un rôle important dans la vie internationale à partir du second mandat d'Eltsine en 1996 et puis ses relations avec ses voisins en Europe centrale et orientale.

En 1997, la diplomatie russe commença à utiliser de nombreux moyens pour s'imposer. Le ministre des Affaires étrangères Evgueni Primakov laissait entendre notamment l'application d'une politique anti-américaine (Riasanovsky, 2014). Afin d'illustrer ce point, on peut évoquer la conférence de l'Association des nations de l'Asie du Sud-Est (ASEAN) cette même année, où le ministre des Affaires étrangers a affirmé que l'Indonésie et la Malaisie pouvaient y prétendre au leadership, en motivant le sentiment antiaméricain de ces pays et en les appelant à assumer un rôle mondial. Primakov s'est rendu en Amérique latine également et a vendu des hélicoptères à la Colombie alors plongée dans la lutte contre le narco trafique au grand mécontentement de Washington. La même année la diplomatie russe a essayé de nuire les Etats-Unis au sein de l'OTAN et de l'Union européenne en créant la troïka ou triumvirat pour ancrer la Russie à l'Europe : Eltsine-Kohl-Chirac ce qui a été célébré dans la presse russe comme " la première victoire presque inconditionnelle de la Russie en politique étrangère » (Riasanovsky, 2014, p. 750). Cet événement surtout symbolique, laisse entrevoir l'ambition du président russe pour faire de son pays un pôle de puissance sur la scène mondiale. Les hommes du Kremlin se sont efforcés pour démontrer à Washington que leur capacité de nuisance était importante.

Un nouvel accent a été également mis sur les relations avec les voisins de la Russie, avec les pays d'Europe centrale et orientale auparavant négligés par Moscou, avec le Moyen-Orient, l'Asie du Sud et l'Extrême Orient. Les axes eurasiens de l'action russe se dessinaient clairement. La conception de cette politique a été formulée en 1998 par Alexandre Doguin, l'un des promoteurs de l'eurasisme. Selon lui, la Russie devait réaliser " un grand espace autarcique " en créant une union douanière eurasienne englobant la Russie, la Biélorussie, le Kazakhstan, le Tadjikistan, l'Ouzbékistan, et la Kirghizie, où pourraient entrer la Serbie, la Grèce, l'Iran, l'Inde, l'Irak, la Syrie et la Libye. Moscou devait contrôler strictement les relations avec l'Occident; instaurer un monopole d'État sur certains sec- 
teurs stratégiques de l'industrie; préférer les relations économiques avec l'Europe et la Chine à celles avec les Etats-Unis et choisir l'euro plutôt que le dollar, en attendant la création d'une monnaie eurasienne.

Moscou s'efforce de consolider son emprise sur la CEI (Confédération des États indépendants : Sadroujestvo Nezavissimykh Gassoudarstv). Le 2 avril 1997, un accord de réintégration a été signé avec la Biélorussie et le 29 août un traité d'amitié avec l'Arménie. Ces accords prévoyaient une alliance militaire entre ces pays et l'instauration de bases russes. La Russie a renoué en outre ses liens traditionnels avec les États " voyous " tels que l'Iran, l'Irak, la Libye et la Corée du Nord (Riasanovsky, 2014).

Il est très important dans les composantes immatérielles de la puissance la représentation de celle-ci par les acteurs et par les opinions publiques. La Russie entendait se montrer dans la scène internationale comme un État qui jouissait encore d'un pouvoir dans le monde, notamment à travers sa politique antiaméricaine. Mais pour que cela soit effectif Robert Frank (2007) suggère qu'il faut une cohésion sociale et politique, fondée sur la façon dont les individus se représentent et vivent ensemble dans une société.

Si l'unité ou la division, la stabilité ou les troubles, la solidité ou la fragilité du régime interne et de ses institutions, renforcent ou affaiblissent la puissance et l'influence à l'extérieur. La puissance technologique et industrielle russe a permis à ce pays d'avoir un grand potentiel militaire moderne et donc un pouvoir de dissuasion en vertu du hard power.

\section{Le potentiel et les forces actuelles militaires de la Russie}

D'après Robert Frank, la puissance militaire constitue également une composante de la puissance, la rangeant dans les aspects du hard power. Il reprend notamment le besoin pour un État à la quête de la puissance de transformer son potentiel en forces actuelles (Frank, 2007). En effet, la force actuelle c'est l'ensemble des moyens principalement militaires et économiques immédiatement disponibles, ainsi que la volonté psychologique. Le potentiel c'est la capacité à se construire rapidement une force plus grande que la force actuelle et la rapidité de cette reconversion. 
Ainsi, il convient d'analyser le potentiel et les forces actuelles militaires de la Russie pour renforcer sa prééminence dans le système international.

L'URSS a représenté l'une des deux grandes puissances militaires du XX siècle. Sa chute en décembre 1991, provoqua toutefois des profondes mutations. La doctrine militaire russe n'est plus adaptée au nouveau contexte géostratégique. Une phase de dégradation de son niveau opérationnel commença à défaut d'investissements. Afin de rattraper ce retard, les dirigeants russes ont engagé des réformes dans le domaine militaire à partir de 1998. C'est à partir du mandant de Vladimir Poutine en 2000 que l'État a commencé à s'intéresser à la réaffirmation de la puissance militaire sur la scène internationale.

La conduite de ce programme de modernisation de la puissance russe a amené à réviser à la hausse le budget militaire qui avait connu une baisse régulière depuis 1988 (Boulanger, 2011). Cette augmentation du budget militaire avait pour objectif de répondre aux besoins de modernisation de l'outil militaire et à faire face à une dégradation de la situation matérielle et sociale des militaires désenchantés et nostalgiques de la splendeur de l'armée soviétique. Ainsi, on passe de 31 milliards de dollars absorbés par les opérations militaires en 1994 à 200 milliards en 2000, soit $2,6 \%$ du PIB. Cette hausse budgétaire a servi notamment à payer les salaires des militaires, les dettes et à répondre aux besoins de maintenance.

On peut voir notamment grâce aux guerres menées en Tchétchénie, région du nord du Caucase en 1994-1996, puis en 1999-2000, le poids de l'armée russe.

En effet, la Tchétchénie est la seule région depuis les accords conclus à Tatars$\tan$ en févier 1994 à revendiquer une pleine souveraineté. À deux reprises, l'autorité russe mena des opérations militaires pour contrôler ce territoire. L'opération de décembre 1994-1996 a conduit à des nombreuses crises militaires, malgré l'envoie de 35000 soldats et en 1999 au nom de la lutte contre le terrorisme, avec une force de 90000 hommes. Cette guerre présente de multiples dimensions : guerre totale pour les Russes au nom de la préservation de l'unité de la Fédération de Russie, guerre de guérilla de longue durée pour les Tchétchènes qui réclamaient leur indépendance et qui l'ont obtenue en 2000, ainsi que le non-respect des droits de l'homme par les militaires russes. Par ailleurs, les effets de cette guerre sur l'armée russe sont considérables. Pertes humaines fortement reprouvés par l'opinion publique et paralysie de la réforme militaire au début des années 2000 (Duroselle, 2012). En définitive, les guerres de Tchétchénie ont mis à l'épreuve une armée en pleine restructuration dans un contexte difficile des relations entre le pouvoir politique et le pouvoir militaire. 
Bien que le redéploiement de l'armée se soit polarisé dans la région du Caucase, il ne faut pas négliger la méfiance qu'ont entretenu les responsables militaires russes vis-à-vis de l'Occident, en particulier l'OTAN. Les progrès croissants en matière de hautes technologies des armées occidentales, à la suite des guerres du Golfe et dans les Balkans ont encouragé les performances militaires russes. Dans les années 2000, cette avance technologique a crée la conviction pour la majorité des officiers russes que l'Occident représentait une menace pour la Russie (Boulanger, 2011).

Néanmoins, il faudrait revenir à l'époque d'Eltsine pour comprendre les mutations profondes de la Russie, longtemps considérée comme sombre, marquée par les crises économiques, sociales et politiques. Il aurait été selon l'opinion publique russe un grand destructeur incapable de redresser le pays : " he was a terminator, not a transformational leader" (Shetvtsova, 2003, p. 10). Cependant, depuis quelques années cette représentation simpliste de la dernière décennie de 1990 commence à être mise en cause. On commence à prendre conscience de la complexité de cette période et des difficultés colossales affrontées par le premier président russe.

\section{Le mandat de Boris Eltsine : L'État en transition}

\section{Les crises politiques internes : 1993-1997}

La disparition du parti-État en 1991 signifiait la fin de la perestroïka comme tentative de rénovation du socialisme. Les deux piliers de la politique de Mikhail Gorbatchev : l'État et le parti se sont effondrés simultanément. La période transitoire a été plus longue et plus instable que dans la plupart des pays ex-socialistes de l'Est. Le mandant de Boris Eltsine voit la naissance d'une nouvelle Russie, qui a dû confronter plusieurs difficultés pour atteindre une relative stabilisation dans le domaine politique, économique et social : " 1991 marked not only the birth of post-communist Russia, but also the stillbirth of its democracys (Shetvtsova, 2010).

Absorbé par les problèmes économiques après son arrivé au pouvoir, Eltsine négligea la réforme politique au début de son mandant. Fin 1991, alors qu’il était en position de force incontestée, il ne dissout pas le Parlement, ne démantèle pas la Première direction générale du comité de sécurité d'État KGB (Komitet Gossou- 
darstvennoï Bezopasnosti) et ne prend pas la décision de faire passer une nouvelle Constitution. Au lieu d'entreprendre la réforme de l'armée et du KGB, il créa en 1992 une structure parallèle. Il a conservé la vielle administration ; la bureaucratie, l'appareil du Parti fédéral restaient en place et pouvaient mener leur guérilla contre le nouveau régime, même dans la sphère du pouvoir (Riasanovsky, 2014).

Le régime transitoire rencontra de nombreux problèmes entre l'exécutif et le législatif. Lé régime soviétique avait toujours reconnu sur le papier la prédominance du Soviet, mais avec l'effondrement du communisme, le législatif pouvait prétendre incarner la souveraineté du peuple (Laran et Regemorter, 1996). Sans doute Gorbatchev fut celui qui a rééquilibré les pouvoirs en revalorisant la fonction présidentielle et Boris Eltsine dans cet aspect l'imita, en recherchant en même temps une légitimité supplémentaire par l'élection populaire. Mais les pouvoirs spéciaux accordés au président par le Soviet suprême étaient temporaires et on a consenti que le président doive obtenir l'aval du Soviet.

Durant cette période d'épreuve de force, Eltsine conservait néanmoins un atout : l'étranger et la majorité de la population considéraient que la fonction présidentielle était la meilleure garantie de l'autorité dans l'État en crise. En avril 1993, il a fait appel au peuple dans un référendum qui était en réalité un plébiscite, puisque les principales questions posées portaient sur la confidence que le peuple devrait accorder au président (53\% lui ont apporté leur soutien et 58,7\% ont approuvé les réformes).

\section{La Constitution de 1993}

Restait la préparation de la nouvelle Constitution. Aucune de deux parties ne désistait pas se remettre à une Constituante. Ainsi, la Commission constitutionnelle du Soviet élabora un projet, mais Eltsine a fait rédiger également par ses conseillers un autre projet constitutionnel, en rejetant tout compromis avec le Parlement. Pour sortir de l'impasse, le 21 septembre 1993, il finit par décider pour un violent coup d'État. Il décida également en octobre la dissolution du Soviet suprême et l'adoption de la nouvelle Constitution, fait par un référendum, le 12 décembre.

La dissolution du Parlement marque la fin de la première étape de la transition postcommuniste. Cette sortie du communisme, certes chaotique et tragique a le mérite d'éviter la guerre civile. Ceci peut s'expliquer par deux facteurs déter- 
minants. L'expérience bolchévique tout d'abord a inculqué aux Russes une profonde désapprobation à l'égard de la violence politique et à promu l'unité pour faire respecter l'ordre public. Le second facteur est l'incapacité des divers groupes d'opposition à monter une vrai organisation, de même que la corruption à grande échelle qui empêchaient l'émergence d'une faction stable, capable de constituer une menace sérieuse pour le pouvoir eltsinien. Ce dernier bénéficiait déjà d'une bureaucratie renaissante et le soutient de ceux qui édifiaient des fortunes (Riasanovsky, 2014).

Tout en proclamant la séparation des pouvoirs que le système soviétique avait toujours récusée, la nouvelle Constitution cédait la suprématie de l'exécutif sur le législatif. Chef de l'armée et de la diplomatie, le président avait dès lors le pouvoir de dissoudre la chambre basse du Parlement appelé cette fois Douma d'État (Gosudárstvennaya Duma). De plus, la chambre haute du Conseil de la Fédération était composée d'une majorité de représentants du pouvoir exécutif, ce qui devait garantir en principe la docilité de cette assemblée.

La nouvelle Constitution jetait les bases d'un régime autoritaire, les mémoires de Gorbatchev nous présentent la dissolution du Parlement et l'adoption de la nouvelle Constitution comme un tournant dans l'évolution du régime créé en Russie après le démantèlement de la RSFSR. Gorbatchev souligne : "Il donna le signal du glissement du pouvoir vers l'autoritarisme. Je n'en fus que d'autant plus surpris de constater que les gouvernements occidentaux justifient - et même approuvaient - cette action barbare, contraire à toutes les normes de la démocratie et d'humanisme " (Gorbatchev, 1997, pp. 869-870).

\section{Les ciments d'un régime autoritaire}

Après le dénouement de la crise de l'automne 1993, le régime eltsinien entra dans une phase de stabilisation. Aucune opposition ne semblait désormais capable de défier le pouvoir. Les affrontements publics cédèrent la place aux compromis. Au sommet, les conflits incessants entre les factions rivales ont permis à Boris Eltsine d'exercer son autorité en tant qu'arbitre. Néanmoins, pour la politologue Lilia Shevtsova (2010) le régime est obligé à faire appel à des groupes d'intérêt régionaux, militaires, bureaucratiques ou économiques pour appliquer ses décisions, limitant son autorite : " the most serious obstacle complicating Yeltsin's democratic leadership was the lack in Russia of influential forces that would try to dismember monolithic power and find a way of the old autocracy" (Brown et Shevtsova, 2001, 
p. 73). À la fin de 1994 le secteur privé est à l’origine de la moitié du PIB de Russie et en 1995 ce pourcentage atteint $55 \%$.

Durant cette période, il y a une marche vers la régionalisation, stimulée par la dislocation de l'économie. Les régions disposant de ressources naturelles abondantes estimaient qu'elles échapperaient mieux aux crises si elles pouvaient s'en réserver les recettes aux dépens du budget général, tandis que la majorité de la population tomba dans la misère.

Les années qui suivent à partir de 1994 sont perçues comme celles de la libéralisation au sens démocratique du terme, mais aussi de la précarité croissante. On remarque qu'au sein des conseils de la FMI, la plupart des prix ont été libérés, déclenchant une inflation qui engloutit l'épargne. La hausse des taux d'intérêt a été forte, tandis que les prix des ressources rares ont été maintenus bas. C'est ainsi que les « oligarques » ont pu acheter le pétrole à bas prix et le revendre à l'Ouest, en faisant des profits considérables.

Mais la production chute de moitié avec des conséquences graves entre 1990 et 1997 . Le rouble dès 1994 s'effondre et la situation s'aggrave jusqu'au krach financier de 1998, privant des ressources non seulement les pensionnés et les retraités, mais une proportion importante de la population. C'est la misère pour une grande partie des couches populaires. Au cours de cette période le PIB a chuté de 60\% (Marcou, 2004). Les institutions politiques n'en fonctionnent mieux, bien que la période d'Eltsine soit officiellement qualifiée de "démocratique " à l'Ouest. À tort ou à raison, l'opinion publique russe avait le sentiment que profitant de l'effondrement de l'Union soviétique, la libéralisation de l'économie conseillée par les Américains a conduit le pays à la ruine.

Cette relative stabilisation du régime se traduit aussi par une régularisation des relations au sein de la Fédération Russe. Si en 1992, le Tatarstan, le Bochkorstan et la Tchétchénie avaient refusé de signer un traité avec Moscou et de payer des impôts au budget général fédéral. Si en 1993, une république de l'Oural avait failli voir le jour, à partir de 1994 les relations avec les provinces "frondeuses " se sont apaisées, à l'exception de la Tchétchénie. Le Kremlin parvient à établir un modus vivendi avec le traité du 15 février 1994, répartissant les compétences du centre et de la république (Riasanovsky, 2014). D’autres traités ont été signés dans les mois suivants avec les autres républiques et régions de Russie. Les élites locales se sentaient plus sûres d'elles-mêmes et n'ont pas été tentés par le séparatisme. En 1995, Eltsine a fait adopter un "statut du service publique », premier 
pas vers la mise en place d'une hiérarchie bureaucratique verticale. Les maires ont été également élus, ce qui a permis à Moscou de rééquilibrer le pouvoir des gouverneurs et de mieux les encadrer. Chaque région possédait un Conseil de sécurité à l'image du centre fédéral (Riasanovsky, 2014). En 1997 est crée au sein du Conseil de sécurité fédéral un département chargé de coopérer avec les Conseils de sécurité régionales.

Tout cela nous suggère que dans l'esprit des dirigeants russes, la normalisation passait par la coopération entre les états fédéraux russes et Moscou, mais aussi par un contrôle des gouverneurs et une maitrise des institutions.

\section{La politique étrangère sous Eltsine}

C'est en 1994, après la dissolution du Parlement par la force et d'adoption d'une nouvelle Constitution, que la Russie commence véritablement à se tourner vers la politique étrangère, car auparavant la lutte pour le pouvoir absorbait toute l'énergie du Kremlin. La Russie achevait de retirer ses troupes d'Allemagne et se préoccupait de trouver un nouvel ancrage en Europe. Lacte fondateur des relations entre la Russie et l'Union européenne a été l'accord du partenariat et de coopération (APC), signé en juin 1994 à Corfou, pour un intervalle de dix ans. Du côté européen, on suppose que la Russie va s'européaniser. En 1994, la Russie est ainsi invitée au G7, en récompense de son adhésion au Partenariat et à un projet de paix proposé par l'OTAN.

Toutefois, la guerre de Tchétchénie éloigna la Russie des Occidentaux pendant cette période. La Pologne, la Hongrie, la République tchèque et la Slovaquie inquiets, insistaient pour être admis à l'OTAN, ce qui troubla les relations des Occidentaux à l'égard de la Russie.

À partir de 1994 s'annonce un tournant dans la politique étrangère russe. Les liens entre la Russie et l'Iran se sont resserrés, ce qui inquiétait les Etats-Unis. Moscou entreprenait par ailleurs accélérer l'intégration de la CEI autour de trois axes: union économique, système de sécurité collective et " espace informationnel commun ". Le 15 avril 1994 est ainsi annoncé la création d'une zone de libre échange englobant douze pays de la CEI et le 20 janvier 1995 est signée une union douanière entre Russie, Biélorussie et Kazakhstan. 


\section{Les caractéristiques de la diplomatie d'Evgueni Primakov}

En janvier 1996, le ministre des affaires étrangères Evgueni Primakov a remplacé Andrei Kozyrev. Cet ancien KGB prenait le contrôle de la politique étrangère en attendant de le faire en politique intérieure (Riasanovsky, 2014). En apparence, la politique de Primakov se plaça dans le prolongement de " coopération " gorbatchévienne avec l'Occident. N'oublions pas qu'à cette époque la Russie avait besoin de l'aide financière occidentale. Primakov s'est particulièrement attaché à éviter une confrontation ouverte avec les Etats-Unis, tout en prouvant à ceux-ci que la capacité de nuisance de Moscou était intacte. Par conséquent, Washington avait intérêt à opter de plein gré pour un partenariat avec la Russie, en laissant à cette dernière la haute main sur les affaires européens et dans l'espace ex-soviétique. La Russie a été obligée toutefois de se résigner à l'élargissement de l'OTAN en 1997, sans obtenir de pouvoir de codécision ni de droit de veto dans les questions de sécurité européenne, et cela en dépit des efforts de Primakov.

On considère que la diplomatie russe a pris sous la direction de Primakov une tournure néo-soviétique de plus en plus marquée. La " multipolarité " primakovienne recouvre la même volonté de nuire aux Etats-Unis sur tous les fronts, de la même manière que chercher à dresser l'Europe contre les Américains ou d'attiser les crises au sein de l'OTAN, comme en témoigne la vente des missiles S-300 à Chypre en janvier 1997. Surtout, il s'est mit en place l'axe Moscou-Pékin pour un "partenariat stratégique » entre les deux pays. De nombreux accords ont été signés afin d'établir entre les deux pays des transferts de technologie nucléaire, l'exploitation des ressources énergétiques et la coopération militaro-industrielle.

Mais, le bombardement de la Yougoslavie en mars 1999, les affaires de corruption des élites russes et la nouvelle guerre de Tchétchénie lancée par le Premier ministre Vladimir Poutine, n'ont fait que nuire les relations entre Moscou et les Occidentaux. En novembre, lors du sommet de l'Organisation de la sécurité et la coopération en Europe (OSCE) à Istanbul, Eltsine vivement critiqué, a boudé le président français Jacques Chirac et le chancelier fédéral allemand Gerhard Schröder et a traité à Bill Clinton de «fils de pute » en claquant la porte. Eltsine a pu vider son cœur car la succession était réglée, il se sentait libre (Baturin, 2001).

La diplomatie russe avait posé des jalons importants à partir de 1994, en prenant une orientation de plus en plus néo-soviétique, mais à cause des crises et de dépendance économique, ce processus s'est ralenti. Ce fut Poutine qui cherchait à fructifier l'héritage d'Eltsine, Lilia Schevtsova le souligne : «In 1993, the Boris 
Yelsin constitution created the framework for a new "personalized power". Yelsin's victory in the controlled elections of 1998 marked an embryonic from of what would later become Russia's imitation democracy" (Shevtsova, 2001, p. 10).

\section{La Russie de Vladimir Poutine : l'affirmation d'un État fort}

\section{Le projet poutinien : une réforme politique interne accélérée}

Pour les présidentielles du mois de mars 2000, Vladimir Poutine s'était fixé quelques priorités : sortir le peuple de sa pauvreté endémique, lutter contre la corruption et la criminalité, sauvegarder la dignité nationale et donner à la Russie une place importante dans les affaires du monde (Marcou, 2004). Il commençait dès lors à prendre aux communistes certains de leurs valeurs.

"La Russie entre dans une période où le pouvoir acquiert le droit moral d'exiger que soient respectées les normes établies par l'État " (Marcou, 2004), déclara le président le 8 juillet 2000, dans son adresse annuelle à l'Assemblée fédérale. Dès lors, il s'est attaqué à la corruption par une réforme fiscale mise en chantier dès l'été de cette même année.

L'économie de marché fut désormais axée sur le social, la société vers une société démocratique et un État de droit. Ce sont les trois principaux aspects que le président proposa pour redresser la Russie. Pour cela, il a œuvré principalement contre les autorités régionales et locales en compétition entre elles. Il voulait ainsi centraliser les politiques et mettre fin aux petits groupes locaux corrompus. Dans ce but, la Russie a été divisée le 13 mai 2000 en sept provinces fédérales dont le tracé était identique aux régions militaires de l'ex-RSFSR. Ces dernières ont été chargées d'encadrer les quatre-vingt-neuf régions russes. Poutine a réduit à sept le nombre des « représentants » présidentiels auprès des gouverneurs. Désormais, ces fonctionnaires ont été chargés de contrôler les administrations locales et de s'assurer qu'elles respectaient les lois fédérales. Tous les organes fédéraux des régions relevaient désormais de préfets misent en place directement par le président : des vétérans du KGB ou des militaires dans la plupart qui ont le dernier mot en politique locale. 
Poutine par cette réforme a restauré l'unité de l'État russe comme il l'avait annoncé, en créant un système de réseaux entre le chef du Kremlin, les patrons des grandes corporations d'État et les leaders régionaux.

La réforme politique avait la même inspiration que celle de la Fédération russe. Comme il s'est attaché à « regrouper » les régions, Poutine a " fusionné » les partis. Le parti Russie Unie (Edinstvo), formé en décembre 2001 est considéré pour Nicholas Riasanovsky (2014) comme une «marionnette du Kremlin actionnée pour la réforme politique ". Le nouveau président russe a eu comme objectif de créer l'unanimité autour du lui, mais aussi contrôler la Douma et donc se débarrasser de toute opposition.

Le processus de concentration que nous venons de décrire s'observe aussi dans l'économie. Les grands groupes furent désormais contrôlés par l'État, qui représentaient une importante partie du PIB national. De plus qu'on assiste à la naissance d'un empire du cuivre, de l'aluminium et d'une industrie d'armements destinés à l'exportation de plus en plus considérable.

Durant le début de son mandat, Poutine est surtout concentré sur la consolidation de sa position et la mise en place d'une série des réformes entre 2000 et 2001. La libéralisation des divises, l'adoption début 2001 d'un impôt sur le revenu uniforme de $13 \%$, introduction d'une loi autorisant la privatisation des terres non agricoles et l'adoption d'un nouveau Code du Travail en décembre 2001, étaient ses principales préoccupations.

Les résultats de la politique économique semble très satisfaisante. Le taux de croissance en 2000 a été de $8 \%$ et en 2001 de 5,5\%. Jusqu'en 2002, la Russie a connu une croissance de rétablissement. Cette période est celle des succès : l'inflation baisse et les investissements sont de plus en plus productifs.

Afin de comprendre la période poutinienne et son succès, il convient d'analyser son idéologie et ses motivations pour placer la Russie au centre des relations internationales.

\section{Une analyse de l'idéologie de Vladimir Poutine}

Le point de départ de l'idéologie de Vladimir Poutine est le rejet du gorbatchévisme et de l'eltsinisme. La malheureuse expérience sous Gorbatchev avait conduit à 
que l'État tombe en pièces. La période eltinienne de son côté, avait laissé un mauvais souvenir aux Russes à cause de l'effondrement économique et du discrédit du pouvoir (Riasanovsky, 2014). D'après Poutine, le régime d'Eltsine avait été au service de l'étranger. La Russie était victime d'un complot international dont les Etats-Unis seraient la force motrice. La démocratie libérale n'était qu'un instrument de cette entreprise pour le démantèlement de l'État russe. Par conséquent, renforcer l'État russe représentait pour Poutine de bloquer l'avancée de la démocratie libérale, à la fois à l'intérieur du pays comme dans l'étranger proche. Ainsi, ce rejet de la période de Gorbatchev et d'Eltsine a conduit à une réévaluation de la période stalinienne et de Staline lui-même.

Son idéologie s'intéresse avant tout à l'unité, qui ne peut être garantie que par une "verticale du pouvoir ", c'est-à-dire une chaine de subordination de l'État calquée sur le centralisme léniniste. Par conséquent, tout ce qui menace l'unité est dangereux, que ce soit le pluralisme des partis, la multiplication des entreprises et des banques, la diversité des religions ou la polyphonie des médias.

Durant ces premières années, la politique étrangère de Poutine se situa dans la continuité de la politique primakovienne. Elle semble suivre les idées du politologue russe Mikhail Deljagin : "Toute lutte pour les intérêts nationaux de la Russie ne peut être que clandestine, la Russie doit avoir recours à la résistance cachée, en s'opposant à "l'agresseur" de manière que celui-ci ne puisse pas découvrir sa main dans les obstacles qu'il rencontre » (Riasanovsky, 2014).

Il nous semble que le but de Poutine est de remettre la Russie au rang de grande puissance et d'assumer un rôle éminent dans tous les domaines, surtout en politique étrangère. En surmontant les effets de l'effondrement de la RSFRS (Marcou, 2004, p. 321), le président considère que « la plus grande catastrophe du XX $X^{e}$ siècle en Russie » s'approche à sa fin.

L'objectif prioritaire du président fut la restauration de l'hégémonie de Moscou sur l'espace ex-soviétique, non seulement pour des considérations de puissance, mais parce que la Russie se sentait constamment menacée par le voisinage de pays qui n'étaient pas soumis à des régimes autoritaires. Ainsi, Poutine relança durant les premières années de son mandat la consolidation autour de la Russie et l'espace ex-soviétique. L'acte fondateur est comme on l'a déjà souligné, la Communauté économique eurasienne (Biélorussie, Kazakhstan, Kirghizistan, Fédération de Russie et Tadjikistan) signé le 10 octobre 2000 (Marcou, 2004). 
Néanmoins, Poutine est conscient que le succès de son projet de restauration de l'hégémonie russe sur l'espace ex-soviétique dépendait de l'attitude des Occidentaux : Européens et Américains.

\section{Le rôle de la Russie dans les évènements du 11 septembre 2001}

Les tragiques évènements du 11 septembre 2001, ont entraîné un tournant dans les relations entre les Etats-Unis et la Russie. Alors que la Russie s'était toujours opposée à l'établissement de bases militaires étrangères près de ses frontières, la politique de Poutine a su s'assouplir face à la menace commune du terrorisme (Marcou, 2004). Ainsi, l'Ouzbékistan avec l'accord russe, accorda des facilités militaires aux Etats-Unis dans leur guerre en Afghanistan. Vladimir Poutine a annoncé également un soutient aux opérations militaires américaines, y compris l'ouverture de l'espace aérien de la Russie. De même, grâce à cette coopération, le Tadjikistan et la Kirghizie ont offert des bases aux forces américaines et le Kremlin échangea des informations avec la CIA.

Le 13 novembre 2001, Vladimir Poutine est reçu à la Maison Blache lors d'une visite officielle, où il a déclaré: "We intend to dismantle conclusively the vestiges of the Cold War and to develop (an) entirely new partnership for (the) long term " ${ }^{1}$. Le président George W. Bush répondait ainsi : "This is a new day in the long history of Russian-American relations, a day of progress and a day of hope. We're transforming our relationship from one of hostility and suspicion to one based on cooperation and trust ". Le président américain a ajouté : "now wève got a friendly relationship".

Au-delà d'une solidarité avec les Etats-Unis, la Russie était aussi en lutte avec les Islamistes, car Poutine était conscient que les combattants tchétchènes furent entrainés en Afghanistan et soutenus par Al-Qaïda. De même, l'État russe n'a pas oublié que l'Afghanistan a été le seul pays au monde à avoir reconnu l'indépendance de la Tchétchénie en avril 2000. Ainsi, Vladimir Poutine avait énormément de raisons pour se solidariser avec les Etats-Unis.

1 Public Papers of the Presidents of the United States: George W. Bush, National Archives and Records Administration, Office of the Federal Register, 2001, p. 1391.

2 Public Papers of the Presidents of the United States..., p. 1392. 
En contrepartie, la Russie a obtenu que la guerre en Tchétchénie passe pour une indispensable guerre contre les Islamistes. Une sorte d'alliance contre le terrorisme a réuni Américains, Russes et Israéliens (Duroselle, 1979, p.622). Poutine espérait que sa bonne volonté permettrait à la Russie à entrer dans l'Organisation mondiale du commerce (OMC) et qu'elle puisse régler la délicate question de sa dette extérieure.

Poutine a porté également une vigilance accrue après les attenants du 11 septembre aux conflits internes de caractère interethnique qui ravageaient certaines anciennes républiques soviétiques. Il s'agissait du conflit azéro-armenien, ceux de la Géorgie avec l'Abkhazie, Ossétie du Sud et l'Adjarie et enfin celui de la Transnistrie entre les roumanophones et les russophones (Riasanovsky, 2014).

Après les attentats du 11 septembre 2001, quand Washington est amené à solliciter l'aide de Moscou pour mener la lutte antiterroriste, la Russie a vu la possibilité de pouvoir retrouver son rôle de "codirigeant " de l'ordre international, objectif qu'elle poursuivait depuis la fin de l'URSS.

En guise de conclusion, le passage du socialisme au capitalisme a été un phénomène unique dans l'Histoire de la Russie et ne pouvait pas se faire sans surmonter diverses crises. La tâche pour Boris Eltsine a été rude, car il a même dû payer de sa propre personne en ce moment des convulsions, crises et troubles. Il a fait de son mieux pour intégrer la Russie dans les institutions occidentales.

Le bilan est complexe, pour le peuple il a été plutôt négatif. Mais l'ancienne superpuissance est devenue un pays émergeant des ruines d'un système qui a été par sa volonté complètement démantelé.

L'héritage légué par Eltsine à Vladimir Poutine est difficile à appréhender. Lorsqu'il a accédé au pouvoir, l'économie du pays était en déroute, le système politique était en chaos et la situation sociale était désastreuse.

On remarque que la Russie poutinienne a connu une rapide ascension dans les affaires internationales. Ceci s'explique par le fait qu'elle a réussi à s'imposer sa volonté auprès des autres acteurs du nouvel ordre mondial. La recentralisation de l'État russe a permis une unanimité politique dont le président est le centre. On remarque également une posture défensive de plus en plus marquée. La Russie est un pays riche que les États étranger rêvent de démembrer afin de pouvoir le piller à leur guise. Derrière ces positions se cache une politique agressive et expansionniste, le pays se sentant menacé par tout ce qu’il ne contrôle pas. Enfin, Pou- 
tine par son excellente expérience de la manipulation des Occidentaux a réussi à imposer ses intérêts au sein de la société internationale. Il se fait " caméléon " à volonté (Riasanovsky, 2014, p.755) en partageant les inclinations de ses interlocuteurs : religieux avec George W. Bush, gaulliste avec les Français et pragmatique et businessman avec les Allemands. L'objectif prioritaire du président Poutine est la restauration de l'hégémonie russe sur l'espace ex-soviétique. Il comptait avec le soutien de l'opinion publique. Un sondage réalisé en 2000 révélait que 55\% des Russes considéraient que la tâche historique de la Russie était de reconstruire un empire successeur de l'empire russe et de l'empire soviétique (Marcou, 2004).

Cependant, il reste en Russie des régions en état de sous-développement. Plus de trente millions de Russes vivent sous le seuil de pauvreté. Cette misère endémique est porteuse de corruption et de criminalité (Kortchagina, 2005, p.221). Pourtant, c'est un État qui tire beaucoup de profit en vendant des matières premières - pétrole, gaz naturel, or, bois, aluminium - mais l'essentiel de l'apport de ces richesses bénéficient d'abord les particuliers, en vertu de la privatisation d'un grand nombre des secteurs économiques.

La population aperçoit Vladimir Poutine comme un homme qui redresse le pays. Si la présidence de Boris Eltsine est restée dans les mémoires comme une période de destruction, la période poutinienne est considérée par la majorité des Russes comme celle du redressement et de la renaissance du pays (Kortchagina, 2005).

Sa réélection le 14 mars 2004 , avec $71,2 \%$ de voix a confirmé la confidence que les Russes ont déposée dans ce dirigeant qui leur a donné la stabilité, le retour à un État fort et une croissance économique stable. À la fin de son premier mandant, Poutine apparaît aux yeux de son peuple comme le reconstructeur de la Russie, celui qui lui rendra sa grandeur et sa dignité.

\section{Bibliographie}

Boulanger, P. (2011). Géographie et géostratégie militaires. Paris: Armand Colin.

Brown, A, et Shevtsova L. (2001). Gorbatchev, Yeltsin, and Putin: Political Leadership in Russia's Transition. Washington: Carnegie Endowment for International Peace.

Cauchy, P. (2008). Dictionnaire de la Russie. Paris: Larousse.

Chaliand, G. (2013). Vers un nouvel ordre du monde. Paris: Seuil.

Duroselle J, et Kaspi A. (2012). Histoire des relations internationales : de 1945 à nos jours, Paris, Armand Colin. 
Duroselle, J. (1979). Qu'est-ce qu'une grande puissance ?. In : Relations internationales (17), 112.

Frank, R. (2012) Pour l'histoire des relations internationales, Paris: PUF.

Gorbatchev, M. (1997). Mémoires, une vie et des réformes, Paris: Rocher.

Public Papers of the Presidents of the United States: George W. Bush. (2001) National

Archives and Records Administration, Office of the Federal Register.

Shevtsova, L. (2010). Lonely power. Washington: Endowment for international Peace.

Thorez, P. (2007). La Russie. Paris: Sedes.

Wackermann, G. (2007). La Russie. Paris: Ellipses.

Renouvin, Pi, Duroselle J. (1991). Introduction à l'histoire des relations internationales.

Paris: Armand Colin.

Laran. M, et Regemorter Van J. (1996). La Russie et l'ex-URSS. Paris: Armand Colin.

Marcou, L. (2004). Les héritiers. Paris: Flammarion, département Pygmalion.

Riasanovsky, N. (2014) Histoire de la Russie : des origines à nos jours. Paris: Robert Laffont.

Shevtsova, L. (2003). Putin's Russia, Washington, Carnegie Endowment for International Peace.

Kortchagina, I, Ovtcharova Lilia, Prokofieva L, Festy. P, et Verger, D. (2005). Conditions de vie et pauvreté en Russie. In: Economie et statistique, (383-385), 219-244.

Vaïsse, M. (2013). Les relations internationales depuis 1945. Paris: Armand Colin. 